\title{
Review on Immobilization of Nanoparticles for Fabrication of Glucose Biosensor
}

\author{
N.F.S.Anuar ${ }^{1, a}$, H. Misran ${ }^{1, b}$, A.Manap ${ }^{1}$, S.Z.Othman ${ }^{1}$ \\ ${ }^{1}$ Nanoarchitechtonic Laboratory, Universiti Tenaga Nasional (UNITEN),Jalan IKRAM-UNITEN, \\ 43300 Kajang, Selangor, Malaysia. \\ aNurulaina@uniten.edu.my \\ b Halina@uniten.edu.my
}

Keywords: Glucose biosensor, glucose oxidase, immobilization, nanoparticles and enzymes.

\begin{abstract}
Glucose biosensor has been improvised from time to time in order to provide fast and accurate detection of glucose concentration especially for diabetic patients. Recently, nanoparticles have rising attention engaging with biosensor application. Nanoparticles serve as the carrier of enzyme immobilization whereas; enzyme is a biological catalyst that reacts with specific substrate in metabolic reaction. Glucose oxidase (GOx) in particular, is the most common enzyme used for fabrication of biosensor. Glucose measurement was done by using amperometric measurement that converted corresponding biochemical reaction between glucose and GOx into electrical output. Response behavior studies were conducted in order to compare the successfulness of GOx immobilization onto GOx-biosensor. Immobilization of glucose oxidase onto nanoparticles can lead towards tremendous impacts especially in new, high sensitivity biosensor.
\end{abstract}

\section{Introduction}

Immobilization of bioreceptors to a surface or template has been improvised from time to time to allow the enhancement of bionsensor's sensitivity especially in limit of detection (LOD) of substrate. Glucose biosensor in particular was developed by using glucose oxidase (GOx) enzymes as bioreceptor that would synthesize the breakdown of glucose into gluconic acid [1,2]. Glucose biosensor is a device used to measure corresponding glucose concentration in human or animal (mammal) using enzyme to generate metabolic process and product as sensing material [1,2]. Biochemical output cannot be measured directly, hence, needs to be converted into measurable output. Transducer acts as the converter in order to convert the biochemical output into readable output such as electric output or visual output.

Fast and high sensitivity glucose biosensor has been developed all the time since glucose is the severe diseases including diabetes, metabolic disorder, kidney failure and heart attack [1]. Nanoparticle provides promising advantages especially in biosensor application. This is due to its small size and high surface area that could increase the amount of enzyme loading [2].

There are two types of enzymes that commonly applied for glucose biosensor which are glucose oxidase (GOx) and glucose-1-dehydrogenase (GDH) [2]. Both of the enzymes are under oxidoreductases category and favor redox reaction of substrate and electron transfer to achieve final product. However both of the enzymes differ in term of rate of reaction, turnover rate, and redox reaction. Glucose oxidase is more widely employed compared to glucose dehydrogenase since GOx is more stable towards extreme ambiances such as temperature and $\mathrm{pH}$, easy to extract, cheap to obtain and favors single phase reaction compared to GDH [1-2].

The reaction shows the GOx catalyzes oxidation of glucose molecule into glunactone (gluconic acid) and hydrogen peroxides $\left(\mathrm{H}_{2} \mathrm{O}_{2}\right)(1)$ [2].

$$
\text { Glucose }\left(\mathrm{C}_{6} \mathrm{H}_{12} \mathrm{O}_{6}\right)+\mathrm{O}_{2} \stackrel{\text { GOx }}{\longrightarrow} \text { Glunactone }\left(\mathrm{C}_{6} \mathrm{H}_{12} \mathrm{O}_{7}\right)+\mathrm{H}_{2} \mathrm{O}_{2}
$$

\section{Nanoparticles}

Nanoparticles are microscopy elements that are ranging from $1 \mathrm{~nm}$ to $100 \mathrm{~nm}[2,3]$. Because of its small size, nanoparticle has high surface area to volume ratio [2]. Reduction of size of enzyme- 
carrier could improve the immobilization of enzyme, thus enhance the higher enzyme loading onto the surface. This unique property also allows the increase of diffusion rate that is essential as template for stabilization of enzyme [3-5]. These are some raising nanoparticles for biosensor application:

Zinc Oxide ( $\mathrm{ZnO})$. Zinc oxide nanoparticle $(\mathrm{ZnO})$ is a metal nanoparticle that contributed in many recent industrial applications including development of sensors and biosensors [6]. It has unique properties such as high isoelectric point (IEP) that is around 9.5 that could attract the attachment of enzyme with low IEP ( 4.0) $[6,7]$. Despite its high IEP, $\mathrm{ZnO}$ is biocompatible and nontoxic that would not harm the attachment of enzyme or other biological component. $\mathrm{ZnO}$ has unique physical properties including one-dimensional hexagonal shape that has four free cations in each of anion. This property is essential to allow electron transfer and promote attachment of functional group of enzyme which is important property for amperometric biosensor. $\mathrm{ZnO}$ has wide band gap energy $(\sim 3.37 \mathrm{eV})$ that make $\mathrm{ZnO}$ a good semiconducting material, which is essential for sensing and transducing component [7].

Graphene. Graphene is a non-metal nanoparticles currently receiving increasing attention of many researchers due to its unique physicochemical properties in many application including sensing material. Graphene is two dimensional nanoparticles thus, very high in mechanical strength and high surface area. Graphene is an excellent semiconductor and sometimes known as superconductor [6]. This property is very important in biosensor application as it could facilitate electron transfer between biomaterial and target substract to perform reaction. Furthermore it can be mass produced and cost effective. Graphene based electrode showed great performance in electrocatalytic activity therefore essential as transducing material for biosensor [6].

Metal Organic Framework (MOF). Metal organic framework (MOF) is a rising, comprise of metal clusters that play role as inorganic joint to connect the organic linker together. MOF provides many promising applications in industries including gas and chemical storages, gas purification and chemical sensing. The coordination of metal clusters with the organic linker formed threedimensional (3D) MOFs by repeating the coordination entities. MOFs materials have unique properties such as high porosity, high flexibility but crystalline framework, low density high surface area and pore volume. These properties are positive for enzyme-carrier for fabrication of biosensor. High porosity and low density could allow maximum adsorption of enzyme onto it hence could stabilize the enzyme immobilization process. The high loading of enzyme could tremendously increase the sensitivity of the biosensor. MOF pore structure can also be functionalized that would allow the electron transfer that is essential for conductivity [6].

\section{Immobilization Methods}

Enzyme immobilization is a procedure of enzyme attachment onto carrier or solid support without losing massive catalytic activity. A good immobilization procedure is crucial to stabilize enzyme for biosensor build up. Immobilization procedure needs to appropriately chosen based on type of enzyme used, transducing method, physiochemical of substrate and material's availability. There are three type of most common enzyme immobilization shown as below:

Cross-linking Immobilization. Cross linking immobilization is achieved when enzyme is crosslinked with intermolecular proteins, either to functionally inert protein molecules, insoluble support matrix or can be cross linked with each other (Fig. 1 (A)). This procedure allows the formation of strong covalent bonding between enzyme and surface of carrier, thus, widely employed in industrial application. However, this procedure can lead to massive losses of enzyme's catalytic activity due to major changes in protein conformation caused by covalent bonding formation in enzyme's functional group [4-5]. 
Adsorption Immobilization. There are two common enzyme immobilization methods using adsorption including physical adsorption and electrostatic interaction [4]. Physical adsorption of an enzyme onto a solid support or a surface is the simplest method to immobilize enzyme. This procedure allows non-specific physical interaction and formation of weak bond (van der waals) bond between enzyme and surface (Fig 1. (B)). This method is easy to perform and only requires minimum steps. Furthermore, this immobilization procedure brings major advantage as it is cheap to perform and tends to less disrupt the protein conformation. For physical adsorption method, enzyme would be deposited onto a surface of solid support for few hours in order to form weak bond. This process needed to be conducted in low temperature to avoid enzyme denaturation [4,5].

Meanwhile, electrostatic interaction is almost as similar to physical adsorption. However, surface of solid support needed to be washed with buffer solution to create hydrophilic surface that could attract the attachment negatively charged enzyme [4,5].

Covalent-bonding Immobilization. Immobilization of enzyme or biomolecule by covalentbonding is one of the widely employed methods apart from cross-linking. An advantage of using this method is the strong covalent bonds formed between enzyme and surface of matrix not allowing enzyme leaching (Fig. 1(C)) [4]. In this method, enzyme will be attached to matrix via functional groups that not crucial for enzyme catalytic activity. The most frequently used covalent bonding formation involves the following side chains of the amino acids; lysine (amino group) and aspartic and glutamic acid (carboxylic group) [4,5]. This method is easy to conduct however; amino residues that involve with catalytic activities must not be involved in covalent linkage to avoid the disruption of enzyme function. Most common supports used for this immobilizations are nonmetal support that can reside bonding with enzyme functional group through amide, ether and carbamate bonds $[4,5]$.

(A)

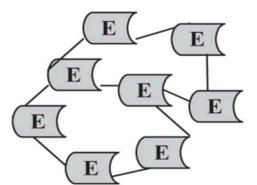

(B)

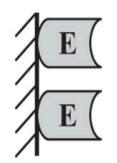

(C)

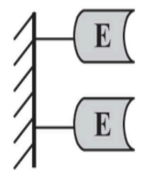

Fig. 1: Enzyme immobilizations: Cross-linking(A), adsorption(B), and covalent bonding (C). E represents enzyme. [9].

\section{Transducing Method}

There are commonly three types of conventional transducing methods in order to convert biochemical reaction between glucose and enzyme which are amperometry, potentiometry and photometry as described below:

Amperometric Measurement. The conversion of biochemical reaction into electric output is called amperometry [9-11]. This method is based on ion exchange in solution. [9]. The reactions are resulted by the redox reaction of oxygen or hydrogen with the impressed voltage from one electrode towards the reference electrode that would produce electron transfer and current output in electrochemical cell [9-12].

Potentiometric Measurement. Potentiometry is a transducing method generally used in order to measure concentration of analyte or solution in electroanalytical chemistry. Usually, the potentials between two electrodes will be measured in the equilibrium state. Therefore, there is no net current and electrochemical reactions occurred during the measurement [9].

Photometric Measurement. Photometric measurement is a measuring method based on the property of light using optical fibers as detector. In this method, the substrate or product that undergoes biochemical reaction will result in light or optical alteration in colorimetric, fluorescent or luminescent. [9]. 


\section{Characterizations}

Most of GOx based biosensors use amperometry as measurement method. [9,10]. The amperometric measurement is based on production of $\mathrm{H}_{2} \mathrm{O}_{2}$ and electron transfer that occurs in the reaction. Glucose concentration is proportional towards production of $\mathrm{H}_{2} \mathrm{O}_{2}$. Thus, the more oxidation occur thus more current produced (Fig. 2 A) [9,10]. The amperometry biosensor was built up using electrochemical cell where voltages needed to be applied in order to facilitate the biosensor. Glucose concentration was measured by the potentials produced. Fig 2 shows the increase of glucose concentration causing the increase in current produced.

Fig. 2 B shows the comparison studies conducted between GOx immobilized onto gold ( $\mathrm{Au}$ ) film modified with $\mathrm{ZnO}$ nanoparticles (GOx-ZnO/Au) (A), GOx immobilized onto Au film (GOx$\mathrm{Au}$ ) (B) and when when only GOx only (C). GOx-ZnO/Au shows better linear line and direct proportional relationship between glucose concentration and current compared to Au film and GOx only. Therefore, the electrode with $\mathrm{ZnO}$ has better sensitivity in for glucose biosensor.

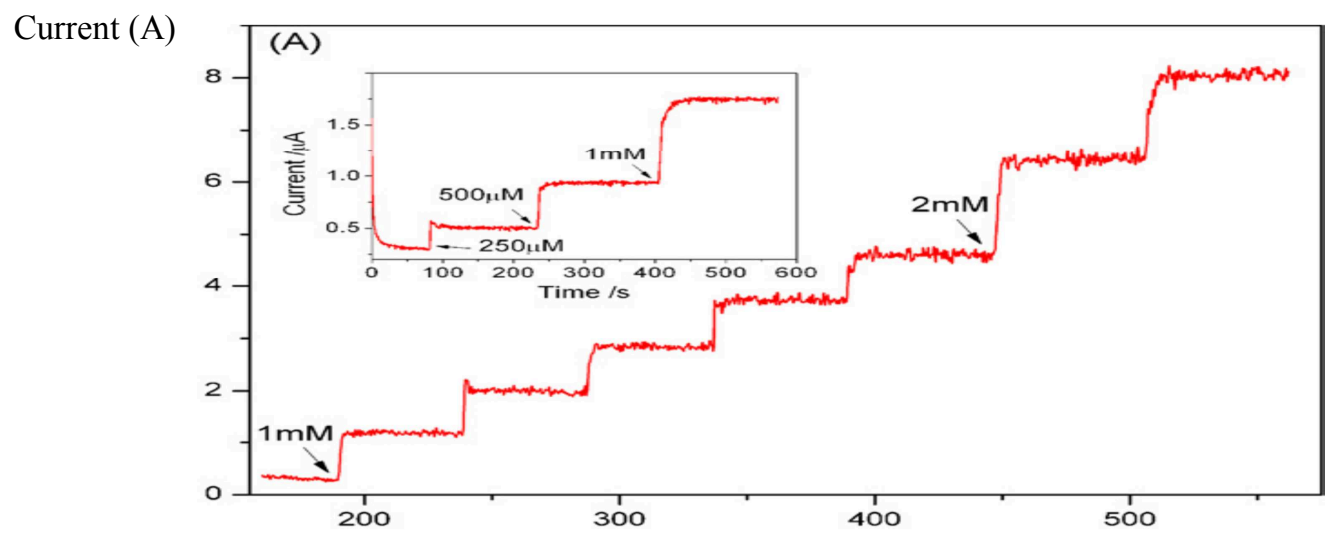

(B)

Current (A)

Time (s)

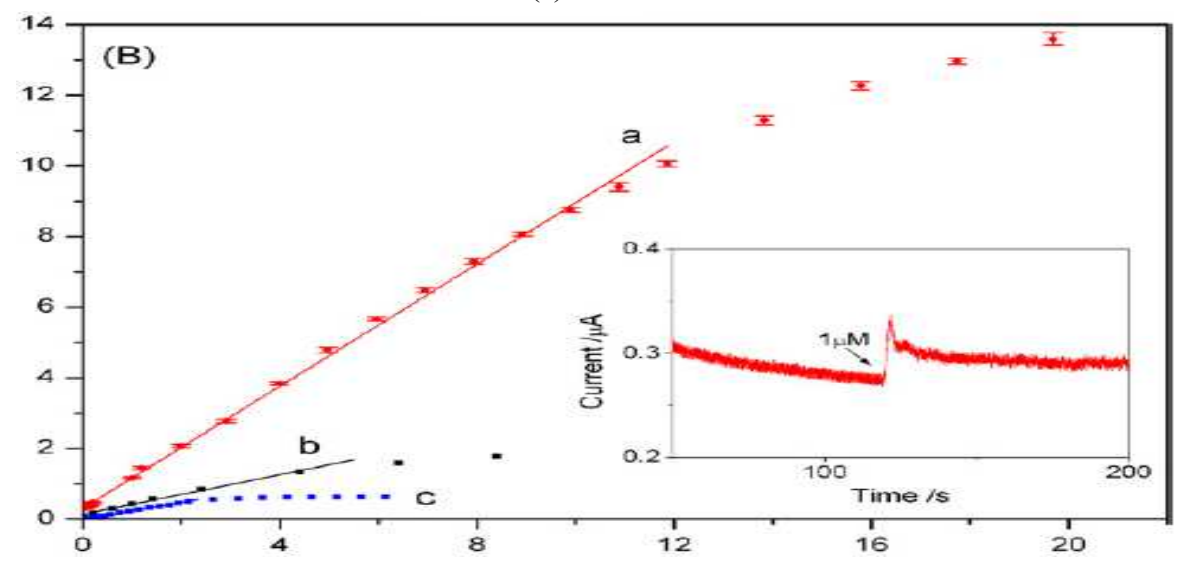

Glucose concentration (mM)

Fig. 2: Current vs. time with increasing glucose concentration (A). Current vs. glucose concentration using $\mathrm{ZnO} / \mathrm{GOx}(\mathrm{A})$, Au film (B) and $\mathrm{ZnO}(\mathrm{C})$.

Environmental Characterization Studies. Enzyme is a biological component hence, very sensitive towards extreme ambiances. From the previous studies conducted, the optimum $\mathrm{pH}$ for $\mathrm{GOx}$ to react was 7 to 7.4 [12-13] and optimum temperature for $\mathrm{GOx}$ activity were between $48^{\circ} \mathrm{C}$ to $50^{\circ} \mathrm{C}$ [12-13]. Experiments were conducted by Kong et. al [7] and Arslan et. al [ 14] in order to measure the optimum $\mathrm{pH}$ and temperature of glucose biosensor. The main objective for the experiments was to examine the existence of GOx properties on the modified electrode in order to prove the successfulness of GOx immobilization onto nanoparticles. Effect of $\mathrm{pH}$ towards glucose biosensor performance was conducted with immobilized GOx-ZnO with constant glucose concentration $(0.1 \mathrm{M})$ and constant voltage supplied $(0.8 \mathrm{~V})$ and varying $\mathrm{pH}$ range from 4.0 to 8.0. 
The current produced was increasing as the $\mathrm{pH}$ value was increased until it reached $\mathrm{pH}$ 7.0-7.3 before it started to decrease at $\mathrm{pH} 8.0[7,14]$. Therefore optimum $\mathrm{pH}$ for GOx biosensor is 7.0-7.3 $[7,12,14]$. Temperature effect experiment towards response of GOx biosensor was also conducted between $20^{\circ} \mathrm{C}(293.15 \mathrm{~K})$ and $65^{\circ} \mathrm{C}(338.15 \mathrm{~K})$. Current was significantly increasing as the temperature increased until it reaches $50^{\circ} \mathrm{C}$ before the current decreased gradiently. It can be concluded optimum temperature for glucose biosensor is $50^{\circ} \mathrm{C}(323.15 \mathrm{~K})$ [7, 14]. The properties withdrawn from the experiments proved that immobilization of GOx onto nanoparticles were successful by possessing out the properties of GOx onto biosensor.

\section{Conclusions}

Three types of potentials nanoparticles for biosensor applications are $\mathrm{ZnO}$, graphene and MOF. Fabrication of glucose biosensor was done by immobilizing glucose oxidase (GOx) onto nanoparticles. The most common immobilization methods applied for fabrication of GOx biosensor were cross-linking, adsorption and covalent bonding. Amperometry, potentiometry and volumetry are the most conventional measurement method for biosensors. Glucose concentration was measured by using amperometric measurement and measuring current produced by oxidation of glucose to produce $\mathrm{H}_{2} \mathrm{O}_{2}$. The increase of glucose concentration would increase the current produced. Response behavior experiments were conducted in order to prove the successfulness of immobilization of GOx onto nanoparticles for GOx biosensor. Glucose biosensor worked optimally with $\mathrm{pH}$ of 7.0 to 7.3 and temperature $50^{\circ} \mathrm{C}(323.15 \mathrm{~K})$.

\section{Acknowledgement}

The authors thank financial support of Ministry of Higher Education under FRGS (FRGS20130104) and ERGS (ERGS09012013).

\section{References}

[1] Y.B. Peng, G. Su, B. Cohan, T. Major, and M. Meyerhoff, Anal. Chem. (2011) 8341-8346.

[2] Z. Zhou and H. Jiang, Pier Andrea Serra (Ed.) (2010) 1-22.

[3] Y. Lin, F. Lu, Y.Tu, and Z. Ren, Nano Lett. (2010) 191-195.

[4] Agrawal, S. Rathore, Gupta, J. Agarwal and Goyal, Diabetes Metab J (2009) 1-5.

[5] Y. Shao, J. Wang, H. Wu, J. Liu, I. Aksay, Y. Lin, Electroanalysis vol 22 (2009) 1027-1036.

[6] A. Sassolas, L. J. Blum, B. D. Keca-Bouvier, Biotechnol. Adv. 30, (2011) 489-511.

[7] T. Kong, Y. Chen, Y. Ye, K. Zhang, Z. Wang, X. Wang, Sensors and Act.,vol B138 (2009) 344-350.

[8] H. Misran, A.M. Aminuddin, F.A.M. Zini, M.J. Ghazali, S. Ramesh, American Institute of Physics, (2010) 277-282.

[9] B. Fang, C. Zhang, G. Wang, M. Wang, Y. Ji,A, Sensors and Act., vol B138 (2009) 304-310.

[10] Y.Lei, X. Yan, J.ing Zhao, X. Liu, Y. Song, N. Luo, Y. Zhang, Colloids Surf., B vol 82 (2010) 168-172.

[11] S. B. Bankar, M. V. Bule, R. S. Singhal, L. Ananthanarayan, Biotechnol. Adv., vol 27 (2009) 489-501.

[12] D. Keilin and E. F. Hartree, Biochem J,42(2) (1948) 221-229.

[13] C.M Wong, K.H Wong, X.D Chen, Appl Microbiol Biotechnol.,78(6) (2008) 927-38.

[14] F. Arslan, S. Ustabaş, and H. Arslan, Sensors, 11(8) (2011) 8152-8163. 